\title{
Effectiveness of various nonpharmacological analgesic methods in newborns
}

\author{
Pancham Kumar, DCH, MD ${ }^{1}$, Rakesh Sharma, MD¹, SukhdevRathour, JR ${ }^{1}$, Sunidhi Karol, $\mathrm{JR}^{2}$, Mohit Karol, DCH ${ }^{1}$ \\ ${ }^{1}$ Department of Pediatrics, Indira Gandhi Medical College, Shimla, India; ${ }^{2}$ Department of Community Medicine, PT. B. D. Sharma PGIMS, Rohtak, India
}

\begin{abstract}
Background: Pain during the developmental period may adversely affect developing neuronal pathways and result in adverse neurodevelopmental, cognitive, and behavioral effects in later life. Immunizations, e.g., hepatitis B vaccine (HBV), administered at birth are painful experiences to which neonates are universally subjected.

Purpose: Here we aimed to study and compare the effectiveness of various nonpharmacological pain management methods in newborns to enable the development of safe and effective analgesic methods for newborns.
\end{abstract}

Methods: This prospective study was conducted at a tertiary care hospital in the Himalayan region. Three hundred term healthy neonates were divided into 6 groups of 50 each. Groups 1-5 were intervention groups, patients of which received a nonpharmacological intervention (breastfeeding, nonnutritive sucking, rocking, $25 \%$ sucrose, or distilled water) before the intramuscular HBV, while patients in group 6 received no intervention. The pain response in each group after the HBV injection was assessed and compared using cry duration and Douleur Aigue Nveau-ne (DAN) score, a behavioral acute pain rating scale for newborns.

Results: Cry duration was decreased in all intervention groups, significantly so in the sucrose (19.90 seconds), breastfeeding (31.57 seconds), and nonnutritive sucking (36.93 seconds) groups compared with controls (52.86 seconds). DAN scores decreased significantly $(P<0.05)$ at one or more points i.e. 30,60 , or 120 seconds in the breastfeeding and $25 \%$ sucrose intervention groups compared with controls.

Conclusion: Oral sucrose and nonnutritive sucking are simple yet underutilized nonpharmacological interventions that effectively reduce pain in newborns.

Key words: Nonpharmacological methods, Analgesia, Newborn infant, Hepatitis B vaccines

Key message
Question: Can nonpharmacological methods be used for neo-
natal pain management.
Finding: Nonpharmacological methods like Sucrose, breast-
feeding etc have shown to significantly reduce the pain caused
by intramuscular hepatitis B vaccination.
Meaning: Nonpharmacological methods are the safe and
cheap potential modalities of analgesia which can be used
during mild to moderate pain in newborns.

\section{Introduction}

Pain is defined as "an unpleasant sensory and emotional experience associated with actual or potential tissue damage. ${ }^{1)}$ Newborns are especially vulnerable to pain and its deleterious effects. Babies have larger receptive fields for nociceptive impulses and possibly a higher density of nerve endings and concentration of substance P receptors. ${ }^{2,3)}$ The density of proprioceptive nerve ending is equal to the adult. ${ }^{4}$ In neonates, the harmful effects of pain include irritability, fear, sense of mistrust towards caregiver, disturbed sleep and wakefulness cycle, delayed wound healing, altered immunological functions, biochemical alterations in energy metabolism. Negative effects on the developing brain also include long-term adverse effects like subtle behavioral changes that may persist up to childhood. Neonates are exposed to various types of painful stimuli in the indoor and outdoor settings. Hence it is very important to have effective tools for the assessment and management of pain. The responses to pain in a newborn are nonverbal which include physiological, behavioral, biochemical, autonomic change and body movements. ${ }^{5)}$ These parameters are used for pain assessment in various scales. ${ }^{6}$

These can be used by the health functionaries to assess pain and evolve strategies to prevent, reduce or eliminate pain. There have been many pharmacological and nonpharmacological methods which are being evaluated in the recent past for effective management of the pain in newborns. The pharmacological

Corresponding author: Pancham Kumar, DCH, MD. Department of Pediatrics, Indira Gandhi Medical College, Shimla, India

घ-mail: panchamdr@gmail.com, https://orcid.org/0000-0003-4338-9450

Received: 5 August, 2017, Revised: 27 July, 2019, Accepted: 13 August, 2019

This is an open-access article distributed under the terms of the Creative Commons Attribution Non-Commercial License (http://creativecommons.org/licenses/by$\mathrm{nc} / 4.0 /$ ) which permits unrestricted non-commercial use, distribution, and reproduction in any medium, provided the original work is properly cited. 
agents like paracetamol, opiates, and local anesthetic agents can be useful for severe pain management. ${ }^{7)}$ While nonpharmacological methods can be effective for the mild to moderate intensity pain relief. Various nonpharmacological methods mentioned in literature nonnutritive sucking (NNS), glucose solution, breastfeeding (BF), kangaroo position, rocking, music therapy, massage therapy, and distilled water (DW). ${ }^{8-11)}$ These methods are low cost, simple and benign and have been found effective. Pain assessment in newborns is a special challenge due to the physiological handicaps of neurological and developmental functions. Various methods used for pain assessment are Neonatal Infant Pain Scale, premature infant pain profile, revised Faces Pain Scale, and Douleur Aigue Nouuveau-ne (DAN). DAN score designed in 1997 is acute pain rating scale in neonates. DAN score is a reliable and valid method of pain assessment in the procedural pain. It is based on the 3 parameters; facial expression, limb movements, and vocal expression. The score is assigned on these parameters depending upon the response of newborn to the painful stimulus. Total of the scores on 3 parameters is used to quantify pain experienced by the newborn.6)

Vaccination during the neonatal period is one of the most common mild to moderate painful experience to which newborns are subjected to universally. ${ }^{12)}$ So it will be worthwhile to find the effectiveness of various nonpharmacologic methods in reducing the pain in newborns during the vaccination. So, we planned this study to find and compare the effectiveness of various nonpharmacological methods in the newborn pain management during the vaccination.

The aims and objectives are as follows: (1) To study the effectiveness of various nonpharmacological methods i.e.; (1) BF, (2) nonnutritive sucking, (3) rocking, (4) $25 \%$ sucrose, and (5) water in reducing the pain in newborns induced during the first dose of intramuscular hepatitis B vaccine. (2) To compare the effectiveness of various nonpharmacological methods i.e.; (1) $\mathrm{BF}$, (2) nonnutritive sucking, (3) rocking, (4) 25\% sucrose, and (6) water in reducing pain on the duration of cry after vaccination and DAN score at 30,60 , and 120 seconds.

\section{Methods}

\section{Cases}

1) Inclusion criteria

(1) Newborns (0-28 days) receiving hepatitis B vaccination.

(2) Parents consenting to take part in the study.

2) Exclusion criteria

(1) Preterm ( $<37$ weeks of gestation) newborns

(2) Sick newborns

(3) Birth weight $<2.5 \mathrm{~kg}$ or $>4.0 \mathrm{~kg}$

(4) Newborns suffering from any major congenital anomaly

(5) Newborns on any drug

This study was conducted in the tertiary care hospital of hilly state of Himalayan region from October 2016 to March
201. Ethical approval from the Institution Ethical CommitteeIGMC Shimla was obtained. Three doses of hepatitis B vaccine are administered at birth within 24 hours of life, 6 weeks and 14 weeks of life under national immunization program in our country (India). A total of 1,697 newborns received hepatitis B vaccine at birth within 24 hours under national immunization program during our study period and 597 among them met the study criteria. Out of 597 eligible newborns, parents of only 300 newborns consented to take part in the study. These 300 newborns constituted our study group. While obtaining consent, parents were explained regarding the various intervention and also explained that their baby could get any of the intervention. Demographic and personal information of each enrolled new. born was recorded as per case record form.

The enrolled neonates were randomized using sequentially numbered, opaque sealed envelopes (SNOSE) method which is an easy, cheap, effective, and reliable method of maintaining allocation concealment, which was maintained by the using sequentially numbered, opaque, sealed envelopes. ${ }^{12,13)}$ Enrolled cases were divided into 6 groups of 50 each using SNOSE method. The person performing randomization was not involved in the study beyond this. The first observer opened one sealed envelope for each baby and recruited that baby to 1 of 6 groups depending upon the group mentioned in that envelop.

Six groups mentioned above along with their suggested mechanism of action are as:

(1) Group 1, BF: Newborns in this group were started on BF 2 minutes before the vaccination and continued until 120 seconds. BF produces analgesia through multisensory stimuli like skin-toskin contact, sucking and glucose present in the breast milk.

(2) Group 2, 25\% sucrose: $2 \mathrm{~mL}$ of $25 \%$ dextrose solution was given through mouth with sterile dropper 2 minutes prior to vaccination. A sweet taste of $25 \%$ sucrose sensation stimulates cortical areas related to the pleasure which helps in the release of endogenous opioids and endorphins which modulate the transmission of painful signals acting on dorsal horn interneuronal gateway regions.

(3) Group 3, DW: $2 \mathrm{~mL}$ of DW was given through mouth with sterile dropper 2 minutes prior to vaccination. It reduces pain by distracting attention and reducing anxiety.

(4) Group 4, NNS: A sterile silicon pacifier (Bonny Baby Care Ltd., Noida, India) was held gently to stimulate sucking. Vaccination was given 2 minutes after the newborn started sucking and it was continued till 120 seconds. NNS decrease hyperactivity promotes calmness and regulates newborns' discomfort.

(5) Group 5, rocking: Newborns in this group were given gentle rocking movement by lifting the head on the palm of the hand. Rocking started 2 minutes before vaccination and continued till 120 seconds. Positioning and tucking reduce pain by distraction, comforting the baby, reducing anxiety and stimulating the vestibular system.

(6) Group 6, none: In this, no intervention was used.

Groups 1-5 were subjected to one or other intervention and 
Table 1. Demographic profiles of the newborns enrolled in the study

\begin{tabular}{llrrrrr}
\hline Group No. & Intervention & Total & Male & Female & Age (hr) & Weight (kg) \\
\hline 1 & Breast feeding & 50 & 28 & 22 & $25.31 \pm 11.01$ & $3.06 \pm 0.425$ \\
2 & 25\% Sucrose & 50 & 29 & 21 & $34.36 \pm 18.08$ & $2.95 \pm 0.427$ \\
3 & Distilled water & 50 & 26 & 24 & $28.58 \pm 26.21$ & $3.08 \pm 0.532$ \\
4 & Nonnutritive sucking & 50 & 23 & 27 & $25.62 \pm 26.84$ & $3.11 \pm 0.573$ \\
5 & Rocking & 50 & 28 & 22 & $23.82 \pm 14.80$ & $2.85 \pm 0.460$ \\
6 & None (control) & 50 & 25 & 25 & $22.31 \pm 11.12$ & $3.00 \pm 0.481$ \\
& Total & 300 & 159 & 141 & $26.66 \pm 18.02$ & $3.00 \pm 0.483$ \\
\hline
\end{tabular}

Values are presented as number or mean \pm standard deviation.

constituted intervention groups while group 6 constituted control group as it was subjected to no intervention.

Newborns were brought to immunization room from the postnatal ward which is around $100 \mathrm{~m}$ from the same, no strict restrictions were imposed to keep the baby nil per orally and most babies were breastfed within 1 hour prior to immunization, with waiting time of about 15 minutes at immunization room. The babies in the BF group were given breastfeed in the immunization room itself during immunization session. Newborns were held in the mother's lap in the warm well-lighted vaccination room. All the newborns were in stage 3 or 4 of alertness and with DAN score of zero before they were subjected to one of the interventions. The intervention decided was given by the same trained health worker. Hepatitis B vaccination administered through intramuscular route at anterolateral aspect of the thigh.

To maintain uniformity and avoid the subjective variation during vaccination the vaccine was administered by the same health worker and needle of the same size $(24 \mathrm{G})$ and same make was used. After vaccination, the following variables were recorded: (1) duration of cry by the second observer; (2) DAN score at 30,60, and 120 seconds after the vaccination by the third observer. DAN score is totally objective based on behavioral assessment, so the point of having interobserver variation seems remote. In addition to that single observer throughout who was familiar with the scoring system will have a more accurate assessment of the score and hence single observer was employed. Single observer, DAN score is pain assessment scale employed in children of $0-2$ years of age. It is based on 3 parameters: facial expression, limb movements, and vocal expression. Each parameter is assigned a score on 3 parameters: facial expression $(0-4)$, limb movements (0-4), and vocal expression (0-3) depending upon the behavior observed. Sum of the 3 scores gives the intensity of the pain. DAN score of 0 signifies no pain perceived and 11 as the maximum.

\section{Statistical analysis}

All calculations were performed using IBM SPSS Statistics ver. 20.0 (IBM Co., Armonk, NY, USA). Continuous variables were expressed as mean \pm standard deviation. One-way analysis of variance (ANOVA) test and student test for continuous variables were used for comparisons between groups. The Pearson chisquare test was used for categorical variables. A $P$ value of $<0.05$ was considered statistically significant. Data were analyzed to
Table 2. Total cry duration of various intervention groups versus control group

\begin{tabular}{llcc}
\hline Group No. & \multicolumn{1}{c}{ Intervention } & Duration of cry $(\mathrm{sec})$ & $P$ value \\
\hline 1 & Breastfeeding & $31.57 \pm 22.26$ & $<0.001$ \\
2 & Nonnutritive sucking & $36.93 \pm 26.19$ & 0.001 \\
3 & Rocking & $51.00 \pm 47.07$ & 0.605 \\
4 & Sucrose & $19.90 \pm 12.84$ & $<0.001$ \\
5 & Distilled water & $38.42 \pm 30.66$ & 0.06 \\
6 & None (control) & $52.86 \pm 48.75$ & \\
\hline \multicolumn{4}{l}{ Values are presented as mean \pm standard deviation. } \\
Boldface indicates a statistically significant difference with $P<0.05$.
\end{tabular}

look for any statistically decrease in pain during vaccination in various intervention groups.

\section{Results}

Three hundred newborns were studied over a period of 6 months. There were 159 males (53.1\%) and 141 females (46.9 $\%)$. The demographic profile of the newborn enrolled in the study under various groups was comparable (Table 1). Comparison of mean duration of cry in different intervention groups with the control group using unpaired $t$ test showed that there was a decrease in duration of cry in all the intervention groups after vaccination in comparison to control group $(P<0.05)$ (Table 2 ). The difference observed in BF, NNS, and 25\% sucrose groups were statistically significant $(P$ value while in rocking and DW groups, the difference was not significant). The difference was statistically highly significant in the group receiving $25 \%$ sucrose and BF $(P<0.000)$. ANOVA was conducted to compare the difference in analgesic effect of various interventions groups namely BF, NNS, rocking, 25\% sucrose, and DW in reducing pain assessed by DAN scores at 30, 60, and 120 seconds. It was observed that there was significant difference of DAN (30 seconds) at $P<0.05$ between different interventions $(F[5,294]$ $=5.049, P \leq 0.000$ ). Post hoc comparison using Tukey honestly significant difference (HSD) indicated that mean DAN (30 seconds) score for sucrose intervention $(3.10 \pm 1.875)$ was significantly different from the DAN (30 seconds) score in BF (4.54 \pm 1.727$)$, NNS (4.64 \pm 1.526$)$, rocking (4.58 \pm 2.139$)$, and control (5.00 \pm 2.283$)$. While ANOVA exhibited no significant effect of DW intervention (3.97 \pm 2.025$)$ on DAN (30 seconds) 
score. Similarly it was observed that there was significant difference of DAN (60 seconds) at $P<0.05$ between different interventions $(F[5,294]=8.8081, P=0.000)$ on post hoc comparison using Tukey HSD mean DAN (60 seconds) score for sucrose intervention $(0.58 \pm 0.8)$ was significantly different from NNS (1.9 \pm 1.5$)$, rocking (2.23 \pm 2.3$)$, DW (1.65 \pm 1.7$)$, and control $(2.82 \pm 2.3)$ while ANOVA exhibited no significant effect when compared with breastfed group $(P>0.05)$ means these intervention are equally effective in reducing pain scores after 60 seconds of painful stimuli. For DAN (120 seconds) scores found to be significantly different in sucrose $(0.05 \pm 0.22)$ and rocking $(1.04 \pm 2.05)$ intervention $(P<0.05)$ while no significant difference was observed with other interventions. DAN score was reduced in all the intervention groups at 30 , 60, and 120 seconds in comparison to control except at 30 seconds for DW group. However, this decrease was significant $(P<0.05)$ only in $25 \%$ sucrose at 30,60 , and 120 seconds and at 60 and 120 seconds in BF group. In intergroup comparison, a significant difference was observed for DAN (30 seconds) at $P<0.05$ between sucrose and other interventions. On post hoc comparison using Tukey HSD indicated that mean DAN (30 seconds) score for sucrose intervention was significantly lower than BF.

Similarly, a significant difference was observed for DAN (60 seconds) at $P<0.05$ between different interventions. Mean DAN score (60 seconds) for sucrose intervention group was significantly lower than NNS followed by rocking and DW group while ANOVA exhibited no significant difference between 25\% sucrose with BF group $(P>0.05)$. Further, DAN score (120 seconds) found to be significantly lower in $25 \%$ sucrose in comparison to rocking intervention $P<0.05$ while it showed no significant difference with other interventions. The pain intensity was significantly lower at 30 seconds in $\mathrm{BF}$ and $25 \%$ sucrose group. Comparison of DAN scores at 30, 60, and 120 seconds in intervention groups with control group have shown statistically significant reduction $(P<0.05)$ at one or more point i.e., at 30 , 60 , or 120 seconds in $\mathrm{BF}$ and $25 \%$ sucrose intervention group when compared with control (Table 3).

Table 3. Mean DAN scores of various intervention groups versus control group

\begin{tabular}{lllll}
\hline Group No. & \multicolumn{1}{c}{ Intervention } & \multicolumn{1}{c}{ DAN $30 \mathrm{sec}$} & DAN 60 sec & DAN 120 sec \\
\hline 1 & Breast feeding & $4.54 \pm 1.72$ & $1.28 \pm 1.31^{*}$ & $0.24 \pm 0.56^{*}$ \\
2 & 25\% Sucrose & $3.10 \pm 1.87^{*}$ & $059 \pm 0.85^{*}$ & $0.05 \pm 0.22^{*}$ \\
3 & Distilled water & $5.00 \pm 2.02$ & $1.65 \pm 1.77$ & $0.63 \pm 1.26$ \\
4 & Nonnutritive sucking & $4.64 \pm 1.52$ & $1.91 \pm 1.55$ & $0.75 \pm 1.38$ \\
5 & Rocking & $4.58 \pm 2.13$ & $2.23 \pm 2.32$ & $1.03 \pm 2.05$ \\
6 & Control & $5.00 \pm 2.48$ & $2.82 \pm 2.38$ & $1.35 \pm 2.06$ \\
\hline
\end{tabular}

Values are presented as mean \pm standard deviation.

DAN, Douleur Aigue Nveau-ne (behavioral acute pain rating scale for newborns).

${ }^{*} P<0.05$, statistically significant difference.

\section{Discussion}

The analgesic effects of various nonpharmacological methods in newborns have been studied in the past and their results are varying. However, there are no comprehensive and definite recommendations for their regular use in day to day clinical scenarios. Our study has been carried out for the same purpose. We have studied the effectiveness of 5 nonpharmacological methods during painful stimulus of HBV vaccination. We conducted our study over a period of months involving 300 newborns divided into 6 equal groups. Groups $1-5$ received 1 of the 5 nonpharmacological methods and constituted the intervention groups while the group 6 acted as the control. Most of these studies done in this regard had quite a less number of the cases as compared to our study. Above this, most of them evaluated 2 or 3 methods in a particular study as compared to our study where we have evaluated the analgesic effect of 5 nonpharmacological methods simultaneously. Gray et al. ${ }^{14)}$ studied the analgesic effect of skin-to-skin contact during heel stick in newborns and found it to be potent intervention in reducing the pain. Carbajal et al. ${ }^{15)} 2003$ studied the effectiveness of BF and a combination of sucrose and pacifier in pain reduction and the result were comparable between 2 groups. Gray et al. ${ }^{16}$ ) found that newborns if put on $\mathrm{BF}$ before, during, and after the heel prick had reduced crying and grimacing and it also prevented an increase in heart rate. Yllmaz et al. ${ }^{17)}$ reported that holding the baby in arms in the upright position decreased the duration of crying during painful stimulus of heel prick. Ozdemir and Tüfekci ${ }^{18)}$ recorded lower pain score and shorter crying duration in response to vaccination using music as an intervention.

Our study has shown that all the intervention groups 1-5 lower mean duration of cry after vaccination as compared to control group. Mean duration of cry in 3 groups namely 25\% sucrose, BF, and NNS was 19.90, 31.51, and 36.93 seconds, respectively which was statically significantly lower as compared to control group which has the mean duration of the cry of 52.86 seconds. The mean duration of cry was lowest in $25 \%$ sucrose group. The difference between BF and 25\% sucrose group was not significant.

The mean duration of cry in rocking and DW group was lower as compared to control but the difference was not significant. On serial observation of the DAN score at 30,60, and 120 seconds after vaccination it was observed that DAN score was lower in all the intervention groups as compared to control group except DW group at 30 seconds. Their results were same as found in our study and both the methods. ${ }^{15)}$ Taddio et al. ${ }^{8)}$ studied analgesic effect of sucrose during various procedures in newborn and found that the effectiveness of sucrose was limited to venipuncture for the newborn and not during the intramuscular vitamin $\mathrm{K}$ injection. Carbajal et al. ${ }^{15)}$ reported the analgesic effect of sucrose and pacifiers during venipuncture and found that the analgesic was even better when both used together. On intergroup comparison in various intervention groups, sucrose was found to be the best as it significantly reduced the pain as shown 
by the serial observations of DAN scores. BF was the secondbest option in pain reduction as it also reduced the pain intensity significantly at all the observations except DAN at 30 seconds. NNS has reduced the duration of cry significantly after painful stimulus but the decrease in DAN scores was though seen but not significant. DW and rocking have shown to decrease the duration of cry and DAN scores but these observations were not significant. Above observations demonstrate that sucrose and NNS are the effective nonpharmacological means of reducing the pain during mild to moderate painful stimuli. Sucrose has shown a better analgesic effect in comparison to BF only at DAN score at 30 seconds. At the rest of the observations, both these interventions were comparable. $\mathrm{BF}$ is a physiological pheno. menon and its great benefits to the newborn, mothers and community on various aspects are well established and beyond doubt. So, it is recommended that BF is one of the effective and useful ways to decrease pain in newborns. During nonavailability of BF other methods like $25 \%$ sucrose or NNS can be employed in the pain management of newborns.

Limitations in our study were as follows: blinding of observer doing DAN scoring and observing total duration of cry was not possible in various groups. Secondly during DAN scoring in the BF group only half of the face was visible to the observer.

Pain has short- and long-term negative effects in newborns. Various nonpharmacological methods are safe, simple, easily available, and effective tools in pain management in newborn. $\mathrm{BF}$ is a physiological phenomenon and along with its all other advantages, can be used as an effective tool to manage mild to moderate pain in newborns and during nonavailability of BF other methods like 25\% sucrose or NNS can be employed. Efficacy of nonpharmacological interventions in reducing mild to moderate procedural pain is already proven in many studies still there are no definite guidelines which method is superior. We comprehensively studied 5 parameters at the same time in contrast to the other studies in past who had studied 1 or 2 interventions. Moreover, we had a higher number of the subjects in each group in contrast to previous studies. In addition to breast feeding hence our findings carry better meanings in the formulation of operational guidelines.

See the Commentary "Prevention and management of pain in the neonatal intensive care unit" in Volume 63 on page 16.

\section{Conflicts of interest}

No potential conflict of interest relevant to this article was reported.

\section{References}

1. Merskey H, Bogduk N, editors. Classification of chronic pain: descriptions of chronic pain syndromes and definitions of pain terms. 2nd ed. Seattle (WA): IASP Press, 1994.

2. Fitzgerald M, McIntosh N. Pain and analgesia in the newborn. Arch Dis Child 1989;64(4 Spec No):441-3.

3. Majcher TA, Means LJ. Pain management in children. Semin Pediatr Surg 1992;1:55-64.

4. Reyes S. Nursing assessment of infant pain. J Perinat Neonatal Nurs 2003;17:291-303.

5. Chiswick ML. Assessment of pain in neonates. Lancet 2000;355:6-8.

6. Lago P, Garetti E, Merazzi D, Pieragostini L, Ancora G, Pirelli A, et al. Guidelines for procedural pain in the newborn. Acta Paediatr 2009;98: 932-9.

7. Anand KJ; International Evidence-Based Group for Neonatal Pain. Consensus statement for the prevention and management of pain in the newborn. Arch Pediatr Adolesc Med 2001;155:173-80.

8. Taddio A, Shah V, Hancock R, Smith RW, Stephens D, Atenafu E, et al. Effectiveness of sucrose analgesia in newborns undergoing painful medical procedures. CMAJ 2008;179:37-43.

9. Campos RG. Rocking and pacifiers: two comforting interventions for heelstick pain. Res Nurs Health 1994;17:321-31.

10. Gradin M, Finnström O, Schollin J. Feeding and oral glucose--additive effects on pain reduction in newborns. Early Hum Dev 2004;77:57-65.

11. Chermont AG, Falcão LF, de Souza Silva EH, de Cássia Xavier Balda R, Guinsburg R. Skin-to-skin contact and/or oral 25\% dextrose for procedural pain relief for term newborn infants. Pediatrics 2009;124: e1101-7.

12. Altman DG, Schulz KF. Statistics notes: Concealing treatment allocation in randomised trials. BMJ 2001;323:446-7.

13. Doig GS, Simpson F. Randomization and allocation concealment: a practical guide for researchers. J Crit Care 2005;20:187-91.

14. Gray L, Watt L, Blass EM. Skin-to-skin contact is analgesic in healthy newborns. Pediatrics 2000;105:e14.

15. Carbajal R, Veerapen S, Couderc S, Jugie M, Ville Y. Analgesic effect of breast feeding in term neonates: randomised controlled trial. BMJ 2003; 326:13.

16. Gray L, Miller LW, Philipp BL, Blass EM. Breastfeeding is analgesic in healthy newborns. Pediatrics 2002;109:590-3.

17. Yılmaz G, Gürakan B, Saatçi Ü. Factors influencing the duration of crying of infants after heel lance. Çocuk Sağlığı ve Hastalıkları Dergisi 2002;45:233-6.

18. Ozdemir FK, Tüfekci FG. The effect of using musical mobiles on reducing pain in infants during vaccination. J Res Med Sci 2012;17:662-7. 